MATEC Web of Conferences 40, 02026 (2016)

DOI: $10.1051 /$ matecconf/20164002026

(C) Owned by the authors, published by EDP Sciences, 2016

\title{
A Method to Analyze Dynamics Properties of Transfemoral Prosthesis
}

\author{
Le Van Tuan ${ }^{1}$, Kengo Ohnishi ${ }^{2}$, Hiroshi Otsuka ${ }^{3}$, Yukio Agarie ${ }^{4}$, Shinichiro Yamamoto ${ }^{1}$, Akihiko Hanafusa \\ ${ }^{1}$ Shibaura Institute of Technology, Japan, ${ }^{2}$ Tokyo Denki University, Japan \\ ${ }^{3}$ University of Human Arts and Science, Japan, ${ }^{4}$ Niigata University of Health and Welfare, Japan.
}

\begin{abstract}
The methodto compute gait cycle forces and moments acting on the hip and knee joints of a lower limb with a trans-femoral prosthesis were investigated. A 3D model of the lower limb with prosthesis was created using CAD software and based on MRI data and real size dimension. The transfemoral prosthesis was modelled as a coupled of links with two revolution joints at hip and knee joint. This coupled link was connected to a bar with translation joint to description the distance walked of people in gait cycle. All parts of the prosthesis were measured and a full-size 3D model was created.The kinematics parameters of a lower limb with a prosthesis were determined from motioncaptured system data. The reaction force was measured with a force sensor in the footplate. The 3D model of the prosthesis was exported to MatlabSimmechanics. The input data which are kinematic parameters were applied to calculate the forces and moments acting on the joints. The results of this study present a method to analyse the dynamic properties of transfemoral prosthesis including speed of the gait. It could be used to calculate the load transferred from the socket to the residual limb. They could also be used to design the structure of a prosthesis and optimize the dynamic characteristics of such a prosthesis.
\end{abstract}

\section{Introduction}

There are many people who have a limb amputated, or who were born with limb different. In US, about 2 million Americans have experienced amputations or were born with limb difference. Another 28 million are at risk for amputation[1]. The rate of amputations caused by traumatic injury and cancer have dropped by around 50 percent over the past 20 years, but unfortunately the rate of amputations due to diabetes and peripheral artery disease is on the rise[2].In other country, especially prevalent in the developing countries and those that have been at war, there are many person have lower limb amputation due to war or bomb and mine remnant after war.

A lower limb prosthesis is designed to replace the functions of the missing lower limb. It mustbe comfortable towear, easytoputonand remove, lightweight, durable, and cosmetically pleasing. Furthermore, the prosthesis must function well mechanically and require only reasonable maintenance. An understanding of the structure's dynamic properties and the load transfer between the socket of the prosthesis and the residual limb is important to the evaluation of the quality of a prosthesis. One of the methods to solve this problem is build the models and simulation to get necessary data.

In some previous studies, models were created to calculate the force and moment acting on the hip and knee joints. XiaohongJia et al used the inverse dynamics based on the Newton's Second Law to calculate the equivalent forces and moments applied at the knee joint during walking [3]. F.Farahmandet almodelled the human body as two dimensional sagittal plane linkage, consisting 8 rigid segments and analysed using rigid body kinematics and inverse dynamics approaches [4]. Mohsen Akbari Shandiz et al used a 2D robotic model of biped locomotion to simulate the entire gait cycle, including the stance and swing phases, of a normal gait and of amputees. The model included seven rigid bodies, these segments were considered to be connected via revolute joints at the hip, knee and ankle[5].

However, almost the models in previous were 2D model, the reaction force was assumed to act on a fixed point on the foot, the position of the center of gravity (CG) of each segment was estimated and not accurate. The model of the socket and residual limb was a pseudo model created in software.

In this study, the authors modeled the residual limb and prosthesis as a coupled links with two revolution jointsat hip and knee joints. This system was connected to a bar with translation joint to represent the distance and speed walked by the subject in gait cycle. There was no relative movement between the residual limb and socket during walking. Each part of the prosthesis was modeled in full size by CAD software. The input kinematic parameters were derived from data acquired with a motion capture system, a footplate force sensor, and mechanical equations. It was exported to simmechanics 
and using the standard Newtonian dynamics to compute dyamics parameters.

The 3D model was build with the real size and it contains 12 partsexpressed fully physical properties and geometry feature. The CG of each part was determined by the software with highly precise. The changingposition of COP was considered during walking of subject and moved to a comfortable point for dynamics calculate process.

These results show the forces and moments acting on the hip and knee joints in the dynamic state including gait speed. They could be used to analyze the knee joint, load impact to socket, and enable the quantitative evaluation of prosthesis. This method offers greater flexibility and the calculation requires less time. The size or material of each part in prosthesis can be change easily. It useful to design or optimization the structure of prosthesis.

\section{Method}

The subject in this study was a man with a right-side trans-femoral amputation. He was aged 47, $167 \mathrm{~cm}$ in height, and weighed $61 \mathrm{~kg}$ without his prosthesis. His prosthesis incorporated a UCLA socket, a Nabco prosthesis, and an Ottobock foot.

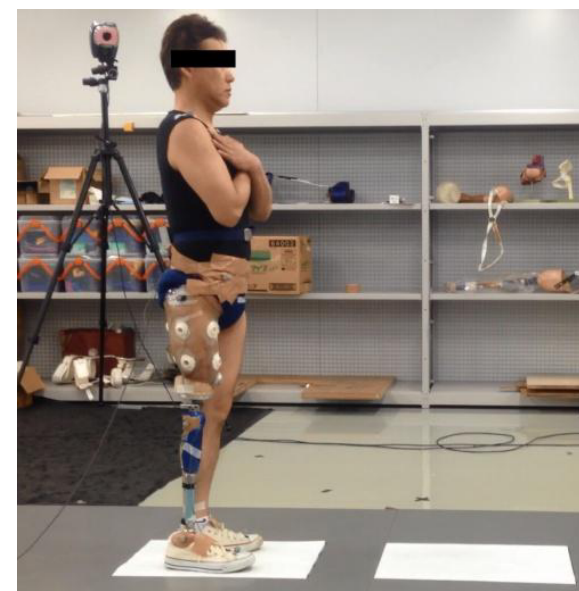

Figure 1. Experiment to get kinematic parameter.

The kinematic data for the lower-limb and prosthesis, as well as the reaction forces applied to the prosthesis foot while walking were measured using a Mac3D system (Motion Analysis Corporation) and a force plate platform (KistlerCorporation). Two plates were placed such that both of the subject's feet struck them during a gait cycle. Data was recorded at a sampling rate of $200 \mathrm{~Hz}$ while the subject was walking (Fig. 1).

\subsection{Established model}

In the Fig. 2 which shows the actual lower limb with the prosthesis, as well as the $3 \mathrm{D}$ model. The $3 \mathrm{D}$ model was created by Creo software. The first link modeling parts in above knee including connected part, socket, resdidual limb. Assumptions were made that there was no relative movement between the residual limb and socket during walking. This link was connected to a hip part by revolution joint which desription the rotation of hip joint. The second link modeling parts below knee including foot, shank, knee joint system. This link was connected to the first link by by revolution joint which desription the rotation of knee joint.
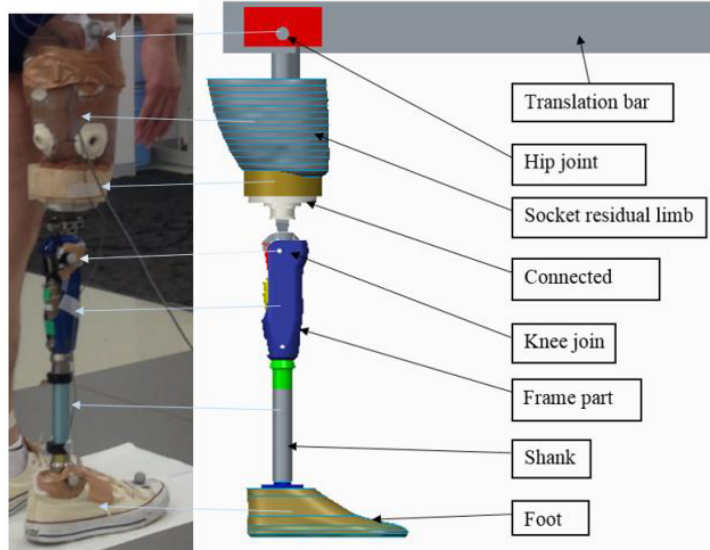

Figure 2. The actual and 3D model of lower limbwith prosthesis

In the first link, the 3D surfaces of residual lim with socket which including bone, muscle, fat, skin and socket were obtained from MRI scans. The MRI scans, that consisted of 17 layers, each separated by $10 \mathrm{~mm}$, were loaded into parallel and contours. After that which were manuallydrawn per each slice and lofted into 3D body by means of a solid modeling software (Creo parametric 2.0 PTC Inc.).

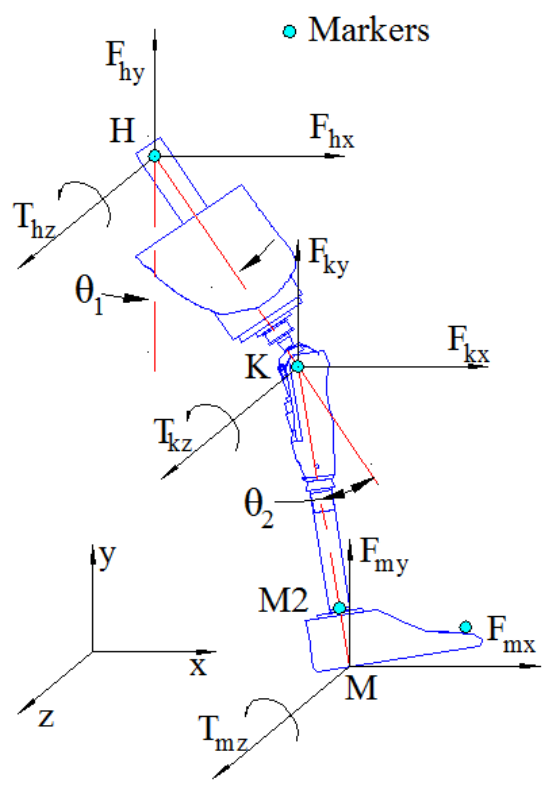

Figure 3. Position of markers and angles on lower limb

The density of the residual limb was obtained by averaging the density of the bone, muscle, fat, skin and socket. The density data of each part in residual limb were reference from research of Ming Zhang et al. [6]. The dimensions of the parts were taken from the actual prosthesis. The material density of each part was calculated from equation:

$$
\mathrm{d}=\frac{m}{V}(1)
$$


Where dis density, $m$ is mass and $V$ is volume of each part.

After that, all parts were connected to translation bar by a prismatic joint at hip part. The movement of this joint represent the distance and speed walked by the subject in gait cycle.

\subsection{Kinematics parameters}

The motion capture system which including seven camera was used to get the kinematic parameter. The positions to which markerswere attached to the lower limb prosthesis are shown in Fig. 3.

The angular rotations at the hip $\left(\theta_{1}\right)$ and knee $\left(\theta_{2}\right)$ joints were defined as shown in the diagram. Matlab (Mathworks Inc.) was used to calculate the angle, angular velocity, and angular acceleration at the hip and knee joints based on the time difference between the markers' coordinates data by cosine rule. The schema movement of transfemoral prosthesis was recognized by makers was show on Fig. 4. and the results of kinetic parameter was show in Fig.6a and $6 b$.

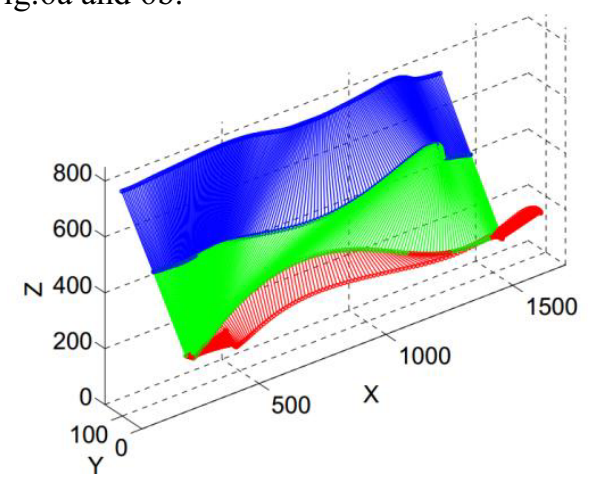

Figure 4. Schema movement of lower limb

The speed of subject in gait cycle was calculated from position data of marker at hip joint. The results are shown on Fig 6c.

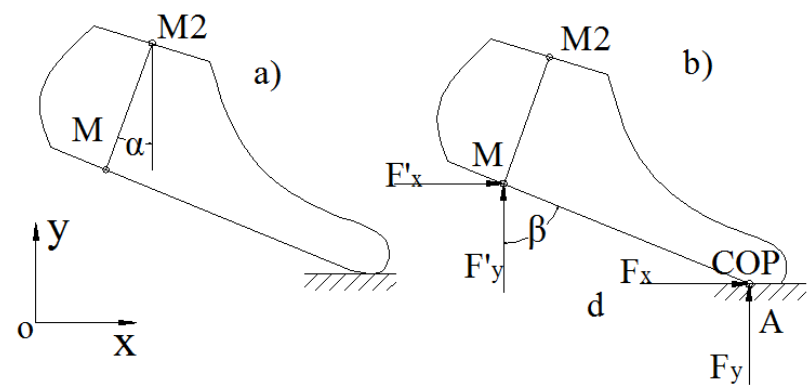

Figure 5. The position of $\mathrm{M}, \mathrm{M}_{2}$ and the shema to calculation the position and the load at $\mathrm{M}$ point

The reaction force acts on the foot of the prosthesis at the center of pressure (COP). The position of the COP in the first force plate coordinate system which corresponds right-side trans-femoral amputation in Fig. $7 \mathrm{a}$ and $7 \mathrm{~b}$. The COP has position change along the below surface of the feet from heel to toe. It need move to a fixed point. In Fig. 5a show the method schema to calculation the position and move the load from COP to M point.

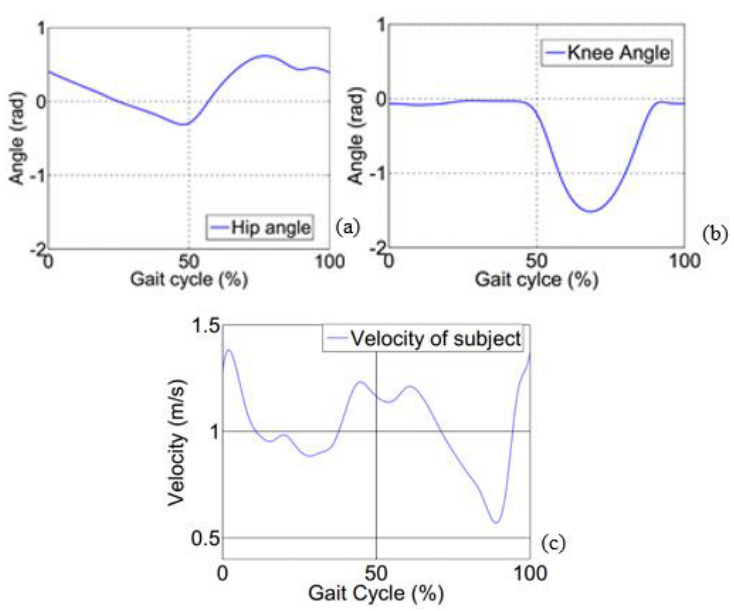

Figure 6. Angle rotation in hip and knee joint and the velocity of patient in gait cycle.

The load wasmoved to point M, shown in Fig.5, have two components. The first component is the force (F'x, F'y) that has same magnitude and direction as reaction force $(\mathrm{Fx}, \mathrm{Fy})$ at $\mathrm{A}(\mathrm{COP})$. The second is a moment with a magnitude defined depends on the positions of $\mathrm{A}$ and $\mathrm{M}$ as equation below.

$$
T_{M}= \begin{cases}F_{y} \cdot d-F_{x} \cdot M M_{2} & \text { when } x_{A}>x_{M} \\ -F_{y} \cdot d-F_{x} \cdot M M_{2} & \text { when } x_{A}<x_{M}\end{cases}
$$

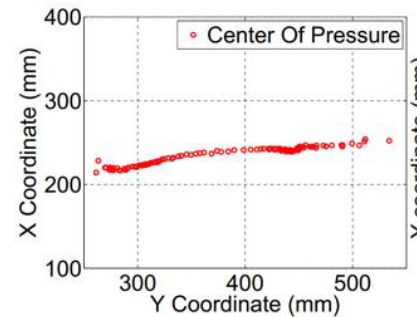

(7.a)

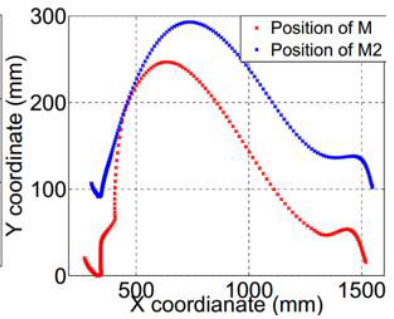

(7.b)
Figure 7. Position of COP on force plate and position of $\mathrm{M}$ and $\mathrm{M} 2$.

The coordinates of $M\left(M_{x}, M_{y}\right)$ were calculated using equations (3) and (4), below. Here, $\left(\mathrm{M}_{2 \mathrm{x}}, \mathrm{M}_{2 \mathrm{y}}\right)$ and $\mathrm{MM}_{2}$ denote the coordinates of M2 and the distance between M and $\mathrm{M} 2$, respectively. The position of $\mathrm{M}$ and $\mathrm{M}_{2}$ are shown on Fig. $5 b$.

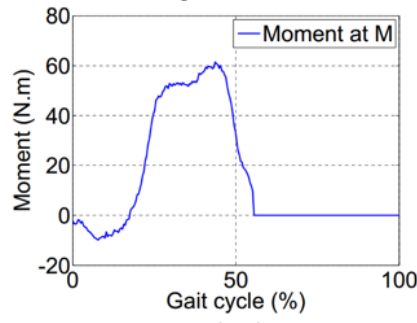

(8.a)

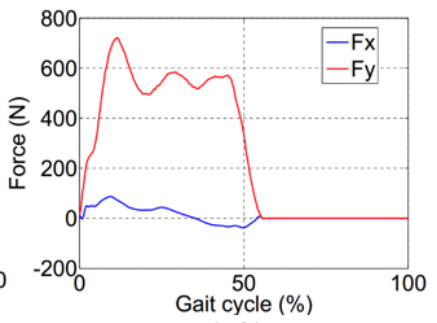

(8.b)
Figure 8. Moment and forces at $\mathrm{M}$.

$$
\begin{aligned}
& M_{x}=M_{2 x}-M_{2} \cos \alpha(3) \\
& M_{y}=M_{2 y}-M_{2} \sin \alpha(4)
\end{aligned}
$$

The momentsand forces at $\mathrm{M}$ were shown in Fig. 8a and $8 \mathrm{~b}$. 


\subsection{Simulation with simmechanics}

The physical data determined with Creo, including the material density, inertia moment, geometry data, and constraints were exported to Simmechanics First Generation (MathWorks). The initial simulation parameters consisted of the angle, angular velocity, and the angular acceleration at the hip and knee joints. The reaction force and moments at the foot were calculated from the measured data.

The block diagram in SimMechanics was shown on Fig.9. The input data (I) include 5 blocks: distance traveled, velocity, acceleration of prismatic joint (Id1) which represent walked distance of patient; angle, velocity, acceleration (Id2) which description the rotation of hip joint; angle, velocity, acceleration (Id3) which represent the rotation of of knee joint; reaction moment at M point (Id4); reaction force at M point (Id5). They were contnectd to joint by joint actuator block (II). The joint actuators supply kinematics parameters to joints.

The 14 physical model of parts and joints that are exported from Creo assembly were in group (III). The blocks express the parts assembly Creo in sequence. The physical, geometry, joint and constrain properties of parts were determined in Creo.The joint sensors (JS) block (IV) that received the output signal are reaction forces and moments at joints and supply them to scope. The result can be observed in the scope or exported to workspace of Matlab.

\section{Results and discussion}

\subsection{Results}

The simulation in simmechanics environment was shown on Fig. 10. The gaitcycle can be divided into two phase: stand phase and swing phase.

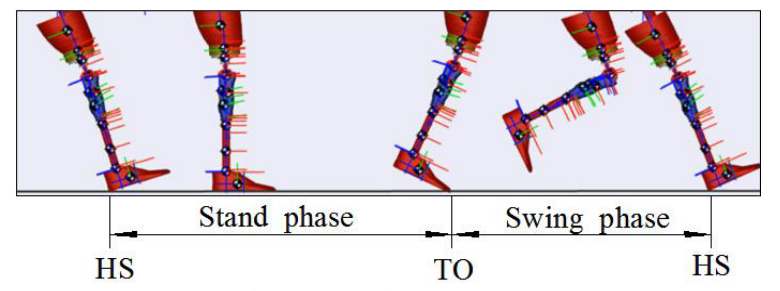

Figure 10.Gait cycle in simulation

The stance phase of gait can counted from the point of initial contact of the foot on to the ground (heel strike HS), the point when the full foot is on the ground (mid stance) and to the point where the the stance phase ends (toe off - TO). The movement of lower limb with transfemoral prosthesis was observed in simulation process. The time and position of prosthesis can be easily to got from it.

The forces (Fx,Fy) and moments (Mz) at the hip and knee joints as determined using Simmechanics are shown in Fig.11. and Fig. 12. They changed according to the gait cycle. The forces start to increase at HS and decrease to minimum at the end of stand phase (TO).

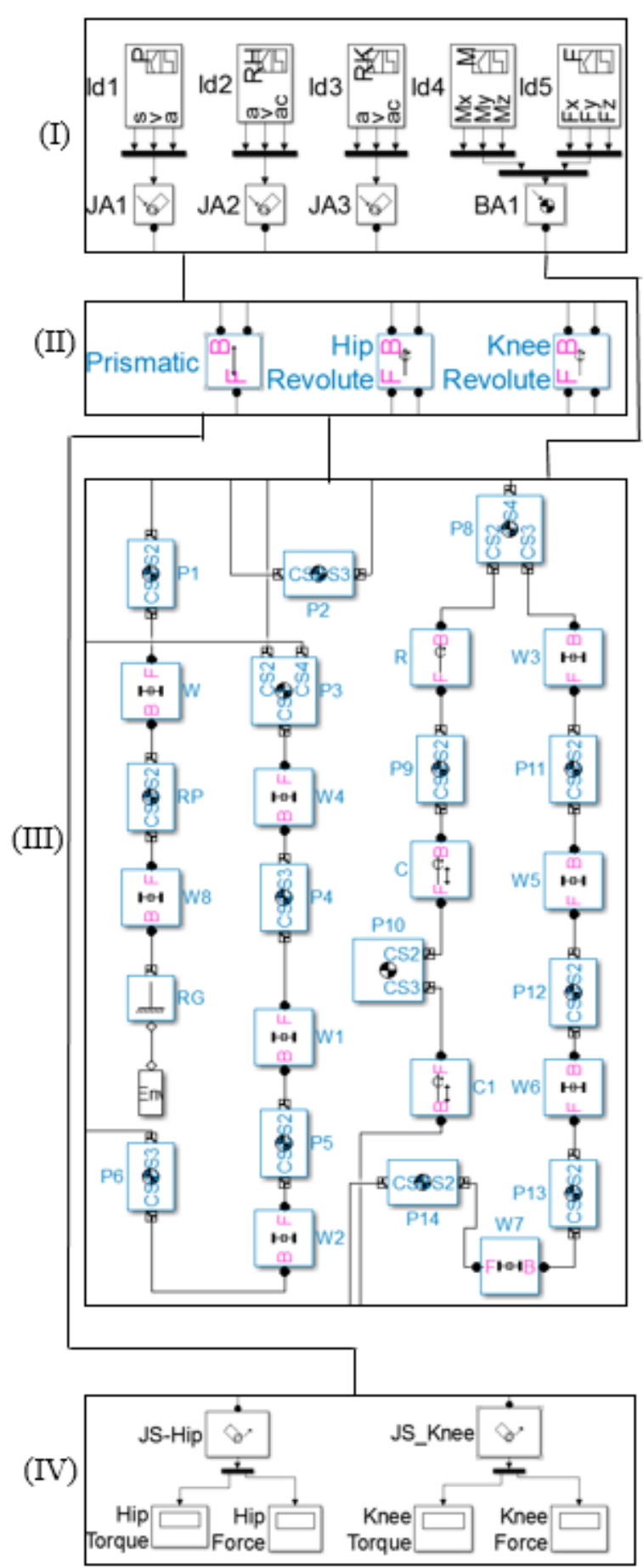

Figure 9. Block diagram in Simmechanics

The graphs of the reaction force at the hip and knee joints are almost the same shape as the ground reaction force. Especially, the magnitude of the reaction force at the knee joint is almost the same as that at the ground.

The direction of the moment at point $\mathrm{M}$, at the hip and knee joints are reversed in stand phase. That are caused the changing of position of COP in foot. The position of COP at the foot was distributed from heel to toe. It determined where ground reaction force impact to prosthesis. In swing phase, the forces and moments at hip and knee joint nearly negligible. 


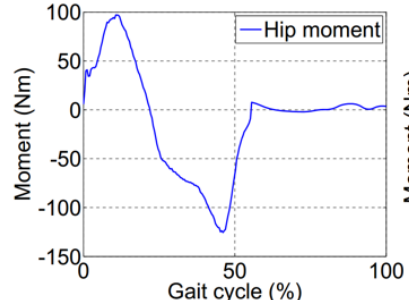

(11.a)

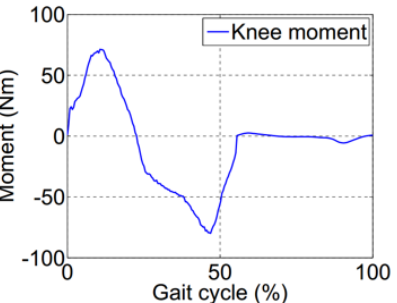

(11.b)
Figure 11. Moments at hip and knee joint

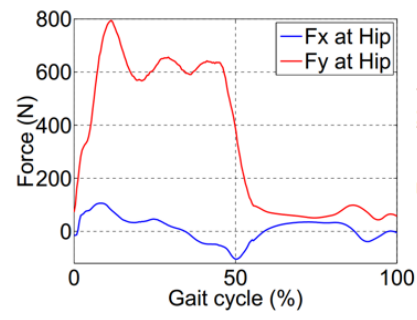

(12.a)

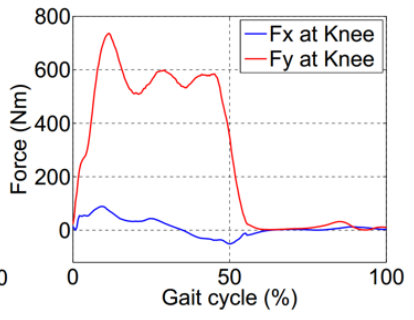

(12.b)
Figure 12. Forces at hip and knee joint

\section{Conclusion}

In this study, a method was built to calculate the forces and moments acting on the hip and knee joints for one gait cycle.This method based on model which describes the movement of lower limb with transfemoral prosthesis nearly in real state accompanied with three movements: walking travelled distance of patient, rotation of hip and knee joint. It was also considered about the ground reaction force and its position in walking.

By this method we can easilyget valueof the forces and moments applied to the hip and knee jointsdespite various changing of parameters of any part in prosthesis. Also we can easily change the shape, material properties, joint type, structure of knee joint, type of foot of prosthesis. The loadswhich computed by this method can be used to calculate the load that affect between the socket and the residual limb.

This model could be used to analyze the knee joint prosthesis, and enable the quantitative evaluation and optimization of the structureof lower limb prosthesis, improve prosthetic design and fitting. It can be combined with other module of matlab, for example simulink, simhydraulic, control system toolbo etc, with various component.

To get more accurate results, this model should be buildmore precise with the shape, material and joint feature. Especially the shape and material of residual limb is important, because determining the shape and material of residual limb is very difficult.

In the future, this study will be enhanced to observe dynamics properties in three plane: sagittal plane, transverse plane and frontal plane. The joint at ankle will be add to the model. The knee joint with pneumatic cyliner will be considered to build a more realistic model.

\section{References}

1. Dr N. Unwin, British Journal of Surgery, 87 (3), pages 328-337(March 2000).

2. http://www.amputee-coalition.org/about-us/

3. XiaohongJia, Ming Zhang, Winson C.C. Lee. Journal of Biomechanics,37, 1371-1377, 2004.

4. F.Farahmand, T.Rezaeian, R.Narimani and P.Hejazi Dinan, ScienticaIranica, 13 (3), pp 261-271, Sharif University, (July 2006).

5. Mohsen Akbari Shandiz, FarzamFarahmand, Noor Azuan Abu Osman and Hassan Zohoor, Int J Adv Robotic Sy, 10, 161,(2013).

6. Ming Zhang, Arthur F.T Mak, V.C Roberts, Medical Engineering \& Physics, 20(5):360-373,(1998). 